\title{
Sub-Nanometer Active Seismic Isolator Control
}

\author{
GAEL BALIK, ${ }^{1}$ BERNARD CARON,${ }^{2}$ JULIE ALLIBE,${ }^{1}$ ADRIEN BADEL, ${ }^{2}$ JEAN- \\ PHILIPPE BAUD,${ }^{1}$ LAURENT BRUNETTI, ${ }^{1}$ GUILLAUME DELEGLISE, ${ }^{1}$ ANDREA \\ JEREMIE, ${ }^{1}$ RONAN LE BRETON, ${ }^{2}$ SEBASTIEN VILALTE ${ }^{1}$ \\ ${ }^{1}$ Laboratoire d'Annecy-Le-Vieux de Physique des Particules (LAPP-IN2P3-CNRS), Université de Savoie, \\ Annecy-le-Vieux, France \\ ${ }^{2}$ Laboratoire SYstèmes et Matériaux pour la MEcatronique (SYMME), Polytech'Annecy Chambéry - \\ Université de Savoie - Annecy-le-Vieux France
}

\begin{abstract}
Ambitious projects such as the design of the future Compact Linear Collider (CLIC) require challenging parameters and technologies. Stabilization of the CLIC particle beam is one of these challenges. Ground motion (GM) is the main source of beam misalignment. Beam dynamics controls are however efficient only at low frequency $(<4 \mathrm{~Hz})$, due to the sampling of the beam at $50 \mathrm{~Hz}$. Hence, ground motion mitigation techniques such as active stabilization are required. This paper shows a dedicated prototype able to manage vibration at a sub-nanometer scale. The use of cutting edge sensor technology is however very challenging for control applications as they are usually used for measurement purposes. Limiting factors such as sensor dynamics and noise lead to a performance optimization problem. The current state of the art in GM measurement and GM mitigation techniques is pointed out and shows limits of the technologies. The proposed active device is then described and a realistic model of the process has been established. A dedicated controller design combining feedforward and feedback techniques is presented and theoretical results in terms of Power Spectral Density (PSD) of displacement are compared to real time experimental results obtained with a rapid control prototyping tool.
\end{abstract}

Key Words: Control, actuator, sensor, piezoelectric, optimization

\begin{tabular}{|c|c|c|c|}
\hline \multicolumn{2}{|c|}{ Nomenclature } & \multirow{2}{*}{$\begin{array}{l}\text { QM } \\
\mathrm{RMS}_{\mathrm{x}}(\mathrm{f})\end{array}$} & \multirow{2}{*}{$\begin{array}{l}\text { Quadrupole Motion } \\
\text { Root Mean Square of signal x in the } \\
\text { frequency range [ } f \infty]\end{array}$} \\
\hline CERN & $\begin{array}{l}\text { European Organization for } \\
\text { Nuclear Research }\end{array}$ & & \\
\hline PSD & Power Spectral Density & $A(s), V(s)$ & $\begin{array}{l}\text { Accelerometer and velocity sensor } \\
\text { transfer function }\end{array}$ \\
\hline CLIC & Compact Linear Collider & $A_{f f}, V_{f f}$ & $\begin{array}{l}\text { FF controllers for accelerometer and } \\
\text { velocity sensor }\end{array}$ \\
\hline
\end{tabular}




\begin{tabular}{|c|c|c|c|}
\hline CMS & Compact Muon Solenoid & $A_{f b}, V_{f b}$ & $\begin{array}{l}\text { FB controllers for accelerometer and } \\
\text { velocity sensor }\end{array}$ \\
\hline FB & Feedback & $F_{a, v}$ & $\begin{array}{l}\text { Extra filters applied to acceleration } \\
\text { (a) or velocity }(v) \text { FF controllers }\end{array}$ \\
\hline FF & Feedforward & $M_{a, v}$ & $\begin{array}{l}\text { Accelerometer and velocity sensor } \\
\text { measurements }\end{array}$ \\
\hline FFT & Fast Fourier Transform & $N_{a, v}$ & $\begin{array}{l}\text { Noise model of the accelerometer }(a) \\
\text { or velocity }(v) \text { sensors, }\end{array}$ \\
\hline GM & Ground motion & $S_{c}$ & Model of the active support \\
\hline IP & Interaction Point & $S_{g}$ & $\begin{array}{l}\text { Transfer function from ground to } \\
\text { support position }\end{array}$ \\
\hline LAPP & $\begin{array}{l}\text { Laboratoire d'Annecy-le-Vieux } \\
\text { de Physique des Particules }\end{array}$ & $S$ & Top support position \\
\hline LHC & Large Hadron Collider & $U_{a, v}$ & Acceleration and velocity commands \\
\hline ML & Main Linac & $W_{n}$ & White noise with PSD $=1$ \\
\hline
\end{tabular}

\section{INTRODUCTION}

The future Compact Linear Collider (CLIC), (CERN collaboration, 2012) currently under study will accelerate electrons and positrons in two linear accelerators over a total length of about $48 \mathrm{~km}$, colliding them at the Interaction Point (IP) with a nominal luminosity of $2 \times 10^{34} \mathrm{~cm}^{-2} \mathrm{~s}^{-1}$. The beam is accelerated and guided thanks to several thousands of accelerating structures and heavy quadrupoles along the Main Linac (ML), see Figure 1. The former accelerate the particles at the required energy, the latter maintain the beam inside the vacuum chamber to reach the required luminosity at the IP.

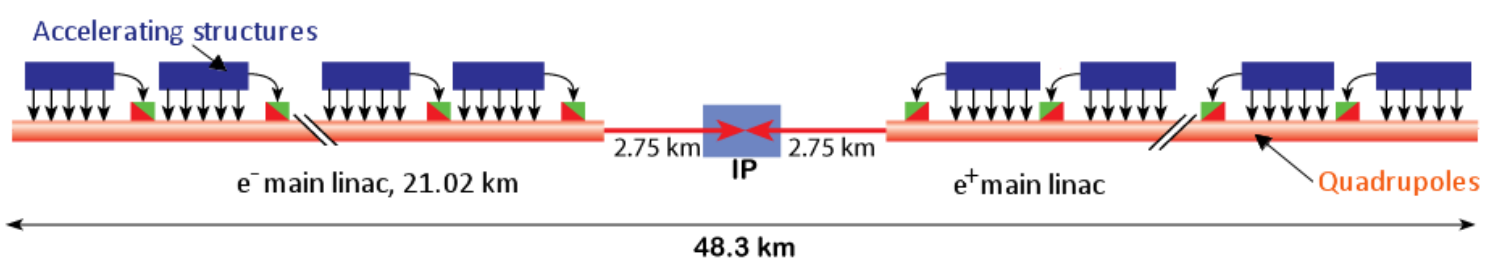

Figure 1. Simplified layout of CLIC.

The luminosity requirement imposes tight constraints on the particle beams' motion and consequently on the Quadrupoles' Motion (QM) subject to Ground Motion (GM). As the shape of the beam is elliptic, its vertical dimension being 45 times smaller than its horizontal one, requirements on the vertical position of the beam are tighter. This paper focuses on the most critical case that is the vertical motion. The desired performances are expressed in terms of displacement RMS (Root Mean Square), which 
is the integral of the Power Spectral Density (PSD) within a given frequency range, as detailed in Eq. 1:

$$
R M S_{x}\left(f_{\text {min }}\right)=\sqrt{\int_{f_{\text {min }}}^{\infty} P S D_{x}(f) d f},
$$

$x$ being the signal to analyze. $R M S_{x}\left(f_{\min }\right)$ is the square root of the power of the signal $x$ calculated in the frequency range $\left[f_{\min }, \infty\left[\right.\right.$. The displacement $R M S_{x}\left(f_{\text {min }}\right)$ specifications depend on the localisation along the accelerator. For the whole Main Linac (ML), $f_{\min }=1 \mathrm{~Hz}$ and $R M S_{Q M}(1)$ shouldn't exceed $1.5 \mathrm{~nm}$. Regarding the IP, $f_{\text {min }}=4 \mathrm{~Hz}$ and $R M S_{Q M}(4)$ should be less than $0.15 \mathrm{~nm}$. Frequency specification $f_{\min }$ is due to beam-based feebacks in the ML (Pfingstner et al., 2011) and at IP (Balik et al., 2011; Caron et al., 2012) able to mitigate only low frequency displacements.

As the future CLIC location site is still unknown, the reference GM is the one measured at LAPP (Annecy - France). This is also the location where the experimental tests were done. However, it is expected that the future accelerator will benefit from better conditions, like the Large Hadron Collider (LHC) (Virdee, 2010) at CERN, safely shielded by 50 - 100 meters of rock below ground. Figure 2 shows the PSD of the GM displacement and $R M S_{G M}(f)$ at LAPP and at the Compact Muon Solenoid (CMS) (The CMS Collaboration, 2008) experimental hall, one of the multi-purpose detectors on the LHC, representative of the detectors of the future CLIC.

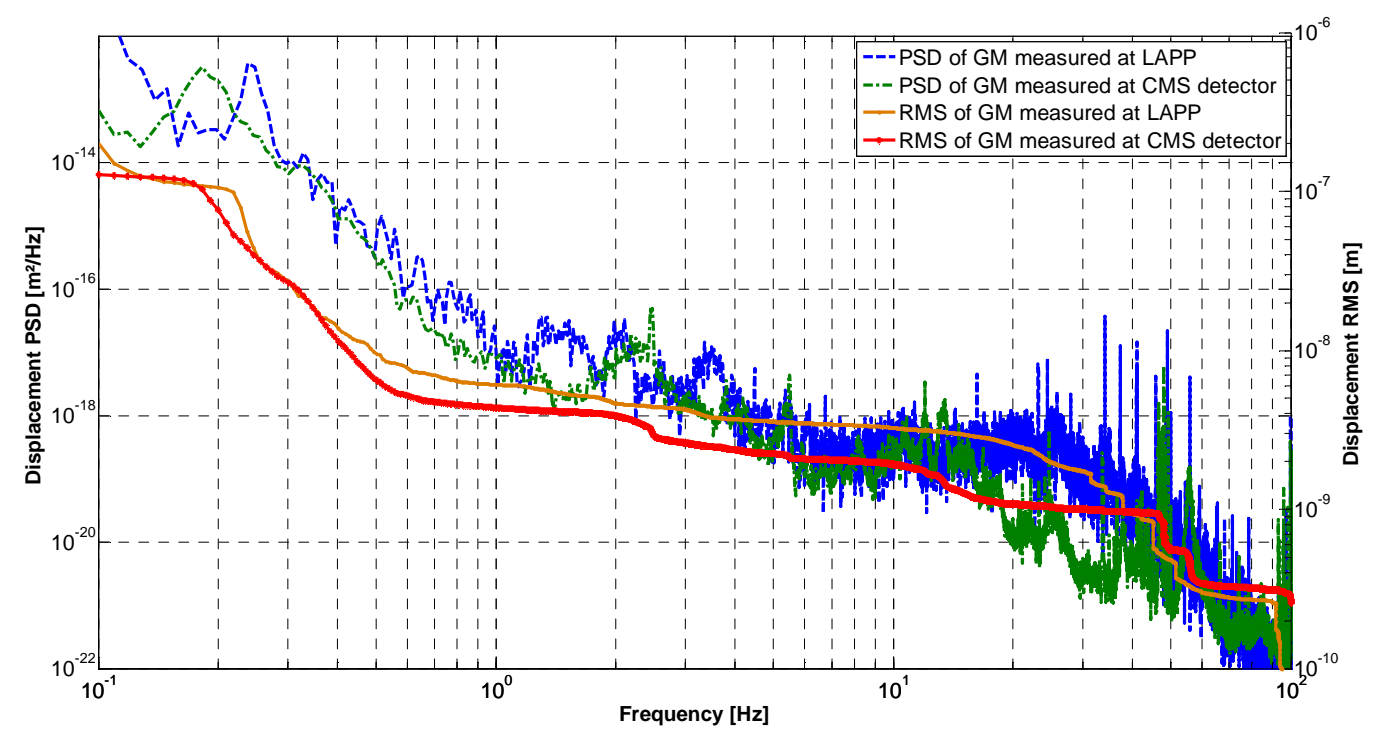

Figure 2. PSD and RMS of GM measured at LAPP and CMS detector.

Before CLIC, such specifications have never been needed for a particle accelerator (or any other system) but in order to meet these tight constraints on the QM, subnanometer active stabilization is envisaged. In precision engineering studies, most 
active controls have been carried out at micrometer scale in many fields (automotive, aeronautic... ) or only positioning, without active control, at nanometer scale (optical system...). It is very exceptional to have active stabilization requirements at nanometer scale, and it is a huge challenge. However, they are necessary for the future particle physics discoveries.

This section summarizes a non-exhaustive list of key experiments built to stabilize the quadrupoles. Table 1 lists some of their characteristics.

Table 1. Summary of current vibration stabilization strategies.

\begin{tabular}{lllll} 
Institution & $\begin{array}{l}\text { CERN } \\
\text { (Collette et al., 2011) }\end{array}$ & $\begin{array}{l}\text { CERN } \\
\text { (Gaddi et al., 2012) }\end{array}$ & $\begin{array}{l}\text { DESY } \\
\text { (Montag, 1996) }\end{array}$ & $\begin{array}{l}\text { SLAC } \\
\text { (Frisch, Chang, et al., 2004) }\end{array}$ \\
\hline Technology & Active & Passive & Active & Active \\
System rigidity & Stiff & Soft & Stiff & Soft \\
Actuator & Piezoelectric & N/A & Piezoelectric & Electrostatic \\
Sensors & Guralp CMG-6T & N/A & Kebe $^{1}$ geophones & GS-1 seismometers ${ }^{2}$ \\
DOF & 6 & 1 & 1 & 6 \\
$R M S_{G M} / R M S_{Q M}$ & 3 at $4 \mathrm{~Hz}, 2.5$ at $1 \mathrm{~Hz}$ & 2 at $4 \mathrm{~Hz}, 0.1$ at $1 \mathrm{~Hz}$ & 4 at $4 \mathrm{~Hz}, 3$ at $1 \mathrm{~Hz}$ & 5 at $4 \mathrm{~Hz}, 3$ at $1 \mathrm{~Hz}$ \\
\hline
\end{tabular}

A performance index defined by the ratio between the displacement RMS of the ground $R M S_{G M}$ and of the quadrupole to stabilize $R M S_{Q M}$ gives the global efficiency of the different experiments at a given frequency.

Although efficient, none has been tested in a quiet environment at the subnanometer scale except the first one (Collette et al., 2011) so the limitations of the whole instrumentation (noise, sensitivity...) is not completely taken into account. Each of these strategies has its own advantages and drawbacks; although the softness increases the isolation at low frequencies, an overall stiff support like in this paper (or in (Collette et al., 2011) and (Montag, 1996)), would be less sensitive against external forces (Artoos et al., 2011). The sensor is one of the most important parts of the stabilization system and should be chosen according to the control strategy. Thus, SLAC $^{3}$ and CERN have built their own sensors (Frisch, Decker, et al., 2004; Janssens et al., 2011), more suitable for an accelerator environment and for control. Another important point, is the number of degrees of freedom (DOF) of the support that determines the ability to control GM vibration in any direction (Collette et al., 2011; Frisch, Chang, et al., 2004). Albeit the developed support has been designed with three DOF, this paper is limited to the control of one degree of freedom.

This paper describes in detail the control strategy applied on a prototype already presented briefly in (Balik et al., 2010) of an active support aimed to reduce the $R M S_{x}(f)$ displacement of ML and IP quadrupoles. Second section provides a technical description of the active support i.e. realistic model of the sensors, actuator, noises and support. Third section explains in detail the control strategy. The experimental setup is described in section 4 and simulation and experimental results are compared. The last section of this paper draws the conclusions and opens to future work. 


\section{ACTIVE SUPPORT}

\section{Electromechanical system}

The most commonly used technology to generate nanometer displacements is a piezoelectric actuator. Although electrostatic actuators (Sarajlic et al., 2003) are sometimes used (Frisch, Chang, et al., 2004) for this type of application, they are not able to reach a sub-nanometer resolution. Moreover, the GM displacement amplitude in the frequency range of interest (i.e. [1 - 100] Hz) can reach $10 \mathrm{~nm}$ (see Figure 2). The actuators also need to support heavy magnets. Thus, the choice corresponding to our need is a PPA10M from Cedrat ${ }^{4}$, resonant frequency: $65 \mathrm{kHz}$, response time: $0.01 \mathrm{~ms}$, $\max$ tensile force: $800 \mathrm{~N}$, max displacement: $8 \mu \mathrm{m}$. The resolution of this actuator is limited by the noise of the driving voltage. To increase the resolution, stiffness is added in parallel with the actuator and decreases the generated displacement (see Figure 3). This solution is suitable because the maximal actuator elongation is 8 times greater than required. By applying the appropriate stiffness, it is then possible to lower the resolution by a factor 5 , leading to a max displacement of $1.6 \mu \mathrm{m}$ and a resolution of about 10 $\mathrm{nm} / \mathrm{V}$.

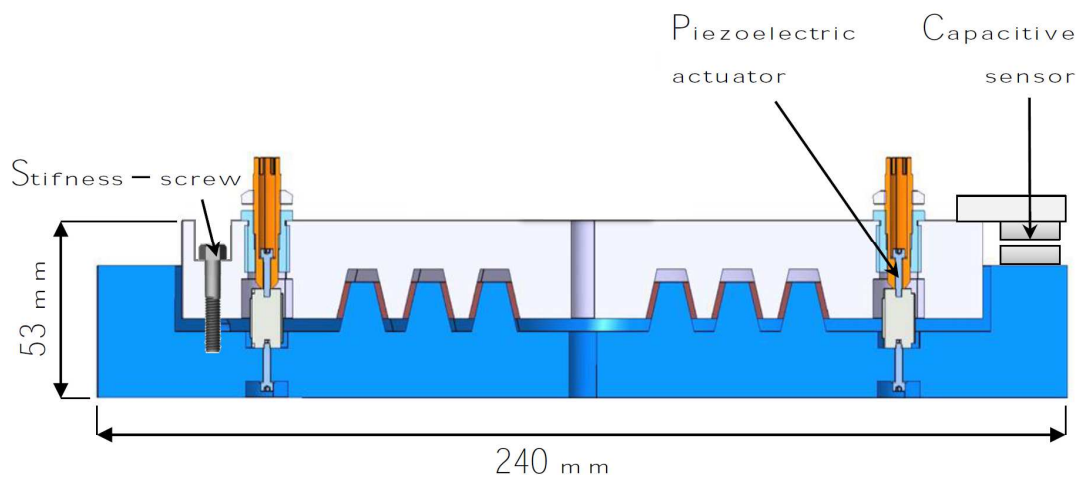

Figure 3. Layout of the active Isolation System with parallel stiffness.

Figure 4 shows the sketch of the proposed active isolation with a quadrupole support. Capacitive sensors are only used for the identification of the model of the mechanical part. The elastomeric strips allow on the one hand the vertical guidance of the upper part of the support and on the other hand, further development with increased number of degrees of freedom, especially for horizontal vibration damping. 


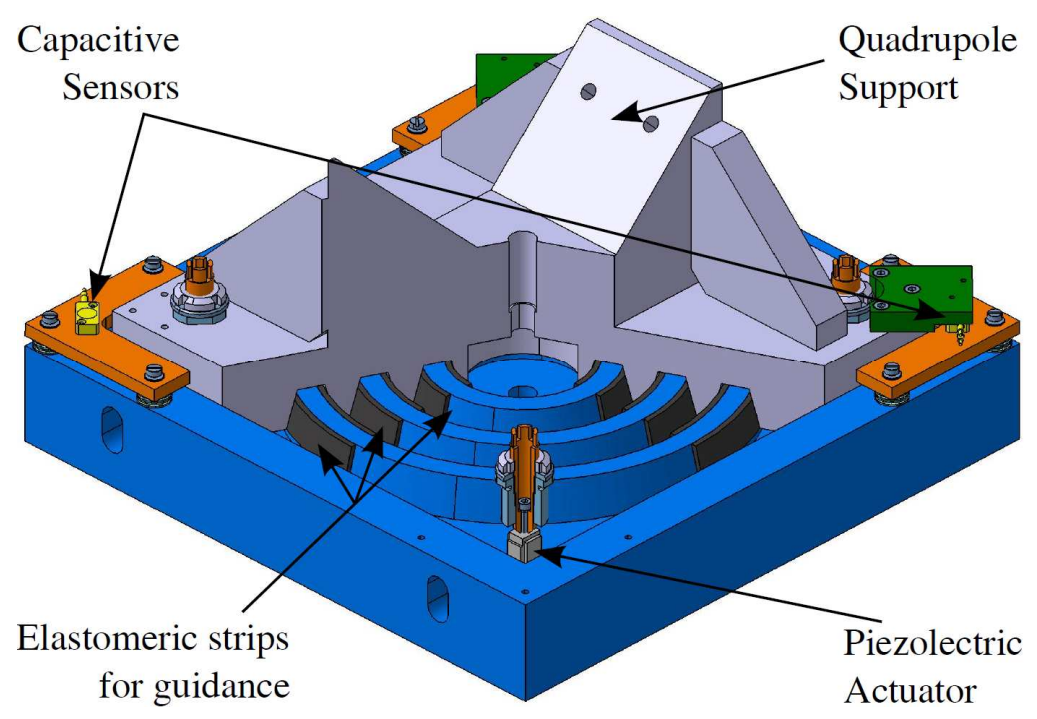

Figure 4. 3D view with the quadrupole support.

Figure 5 represents the frequency response $S_{c}(s)$ of the active support from control command to the support position. The gain corresponds to the piezoelectric actuator sensitivity of $10 \mathrm{~nm} / \mathrm{V}$.

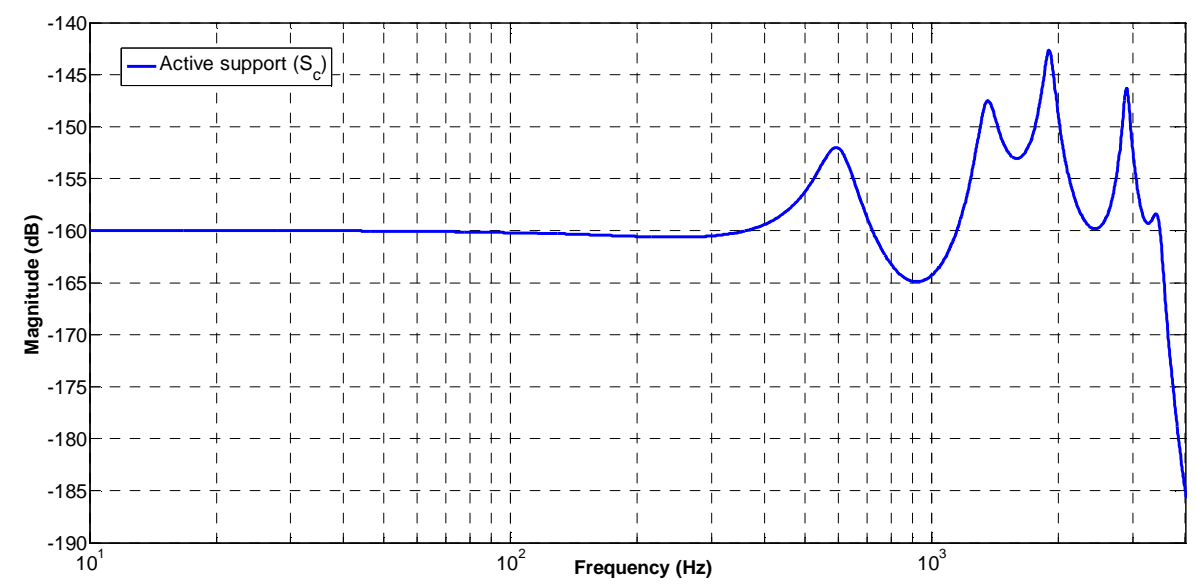

Figure 5. Frequency response of the active support $S_{c}(\mathrm{~s})[\mathrm{m} / \mathrm{V}]$.

The transfer function from ground to support position $S_{g}(s)$ has the same dynamics but with a unity gain (Balik et al., 2010). In the final experiment, each IP quadrupole will be installed on 5 supports. In this paper the control of one support without load will be tested as the design of the quadrupole is not definitive and a representative prototype is not yet available. 


\section{Sensors}

Beam components like quadrupoles have to be stabilized down to the subnanometer level. The vibration isolation is a problem that has led to many approaches (Preumont et al., 2002; Tjepkema et al., 2012), but sub-nanometer stabilization requires state of the art electronic devices such as very low noise sensors, high resolution actuators or an ultra-low noise acquisition chain.

The use of cutting edge sensor technology is very challenging for control applications as they are usually used for measurement purposes. It induces complex management of the given sensor transfer function with limited bandwidth, spurious frequencies, delays...For these reasons, two types of commercial sensors are used in this paper for the measurements and controls: the velocity sensor Güralp CMG-6T for the low frequency range and the acceleration sensor Wilcoxon 731A for the upper frequency range, despite its internal delay. The experimental transfer function of the velocity sensor $V(s)$ and the accelerometer $A(s)$ are shown in Figure 6.

The Güralp sensors are high sensitive electromagnetic geophones measuring velocity in 3 directions (vertical and 2 horizontal). They have a flat frequency response from $0.03 \mathrm{~Hz}$ to $100 \mathrm{~Hz}$. The operating range is yet closer to [1.5 - 90] Hz as their internal noise at low frequency is rather high when the ground velocity is very low. Wilcoxon sensors are high sensitive piezoelectric accelerometers measuring in the vertical direction with a flat frequency response between $0.01 \mathrm{~Hz}$ and $500 \mathrm{~Hz}$, but with an operating range of $[10-200] \mathrm{Hz}$ due to the noise.
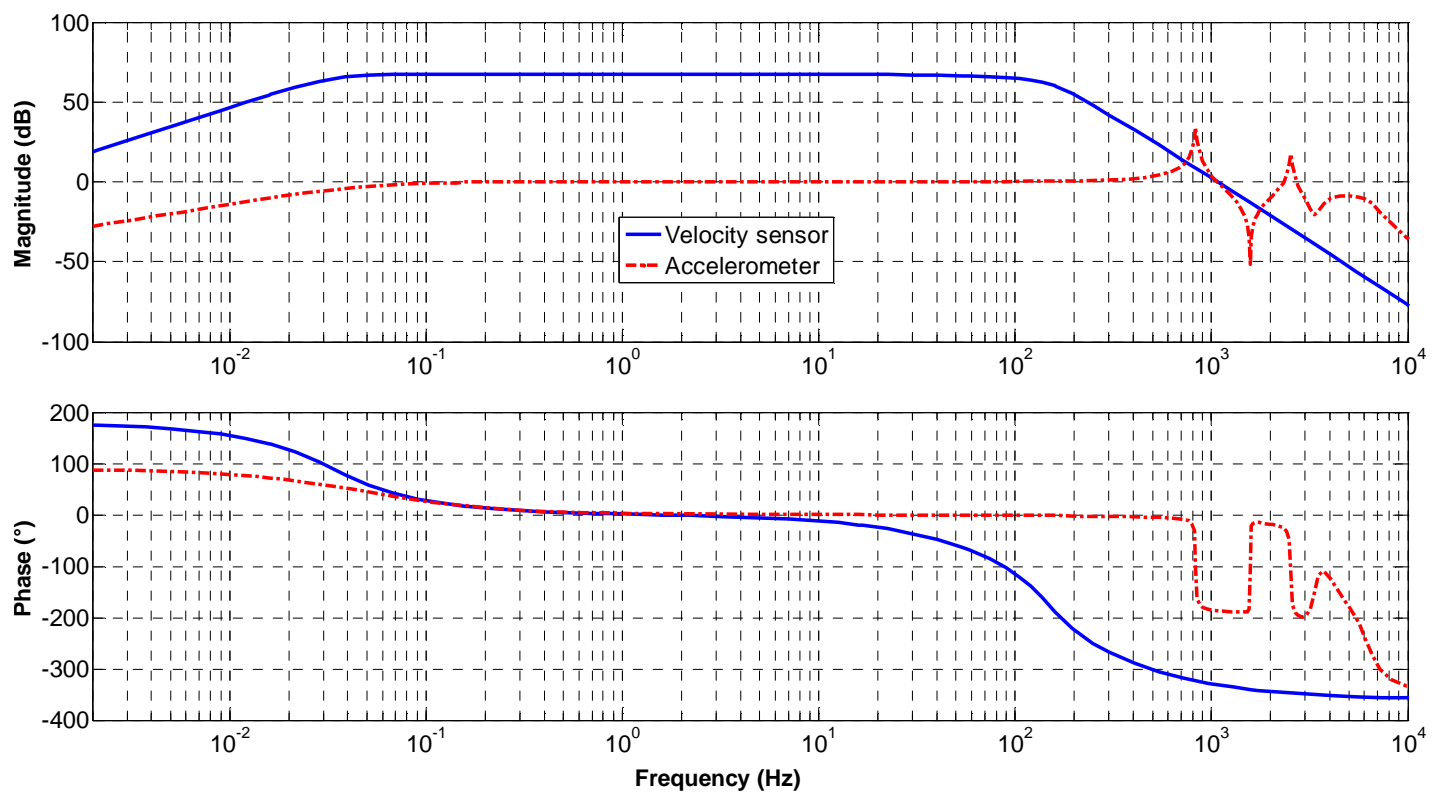

Figure 6. Velocity sensor and accelerometer transfer functions. 
When measuring nano-displacements, resolution and noise of the measurement chain is also a limiting factor. Consequently, these noises and those of the sensors have been measured and are described in the next section.

\section{Acquisition chain, sensors and analog converter noises}

The Analog to Digital Converter (ADC) as well as the Digital to Analog converter (DAC) (respectively ds2004 and ds2102 from dSPACE, compatible with Matlab/Simulink) are high-speed, 16 bits resolution boards. The noise of these converters has been characterized as shown in Figure 7.

Signal conditioning is done thanks to active high-pass and low-pass filters and amplifiers from Krohn-Hite Corporation. The Krohn-Hite Model 3384 has four independent channels and provides a tunable frequency range from $0.005 \mathrm{~Hz}$ to 200 $\mathrm{kHz}$. The level of noise of this system has been measured and is negligible compared to the ADC' noise in the range of interest (i.e. [0.1 100] Hz).

The performances of the measuring system (including sensors) have been characterized by the data that were taken with the sensors of the same model placed side-by-side. The sensor's noise is then calculated by using the corrected difference method (The NLC Design Group, 1996). Results in terms of PSD are given in Figure 7.

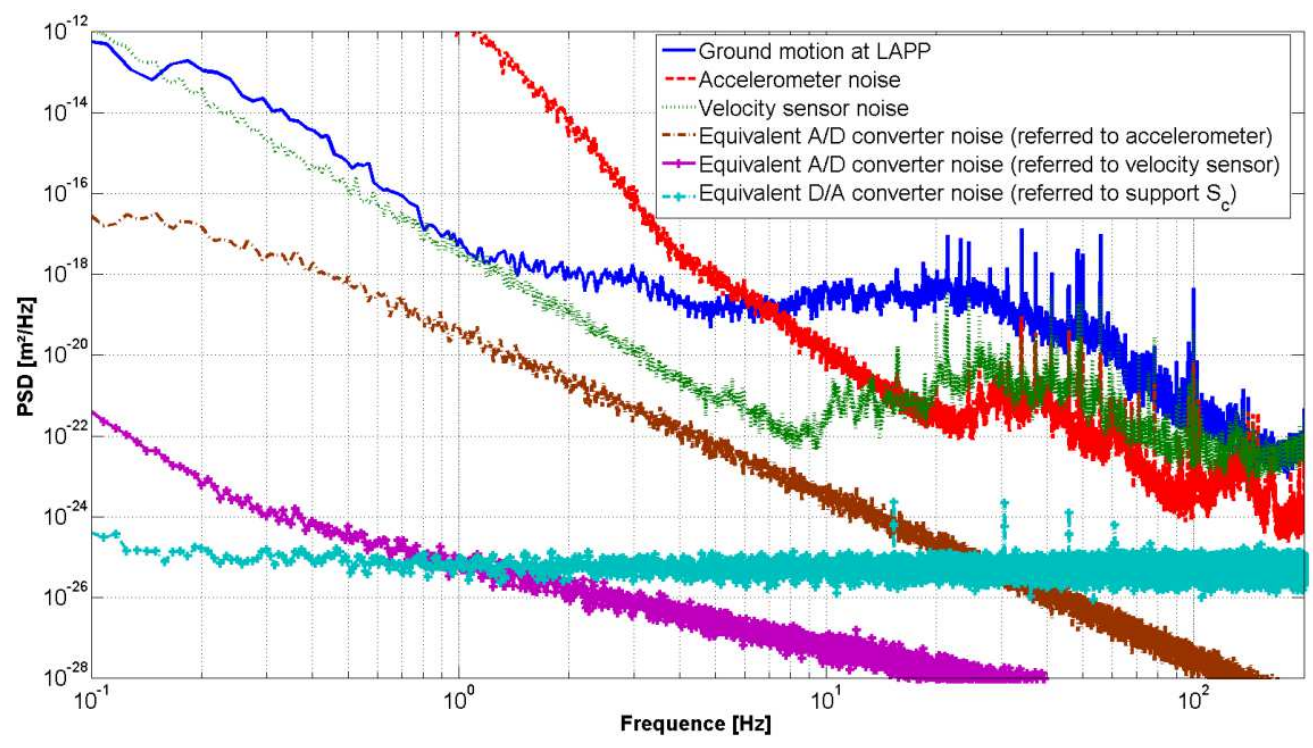

Figure 7. PSD displacement equivalent noise of sensors, D/A and A/D converters compared to ground motion

All these noises have been measured using the test bench presented in Figure 13. In order to compare their respective influence, they are all expressed in $\mathrm{m}^{2} / \mathrm{Hz}$. For that, the corresponding transfer functions (inverse of sensors, amplifiers and actuators) have been used. 


\section{Noise models}

The sensors' models are driven by a white noise $\left(W_{n}\right)$ with a PSD equal to 1 whatever the frequencies. In order to fit experimental results, the transfer functions of the noise models for the accelerometer and the velocity sensors were found to be:

$$
\begin{gathered}
N_{a}(s)=\frac{0.02 s+1}{1.06 s+1} 2.13 \times 10^{-4}, \\
N_{v}(s)=\frac{1.866 \times 10^{-11} s^{3}+1.829 \times 10^{-9} s^{2}+6.897 \times 10^{-8} s+10^{-6}}{2.533 s^{3}+2.546 s^{2}+s} \widehat{V}(s),
\end{gathered}
$$

where $\widehat{V}(s)$ is the identification of the transfer function of the velocity sensor.

The ADC noise is modeled by $W_{n}$ multiplied by a gain equal to $1.42 \times 10^{-6}$ corresponding to a $97 \mathrm{~dB} \mathrm{~S} / \mathrm{N}$ ratio at a sampling period of $50 \times 10^{-6} \mathrm{~s}$ while the D/A converter noise is modeled by $2 \times 10^{-6} W_{n}$ corresponding to a $94 \mathrm{~dB} \mathrm{~S} / \mathrm{N}$ ratio.

\section{CONTROL STRATEGY}

The objective of the control strategy is to reject GM. The control strategy has to take into account:

- noises of sensors all through the acquisition chain,

- noise of digital to analog converter,

- sensor and support transfer function characteristics.

The GM can be measured on the floor and on top of the support; the control strategy will then use 4 measurements coming from 2 accelerometers and 2 geophones.

\section{Control scheme}

FF control is best deployed in control systems design applications where the process and the disturbances are well understood. This is indeed the case, on the one hand, for the active support and the sensors which can easily be characterized and on the other hand for the GM which can be measured. Due to the limited but complementary characteristics of the sensors, the operating range of the FF control can be extended by using both sensors. Figure 8 represents the block diagram of the FF control. 


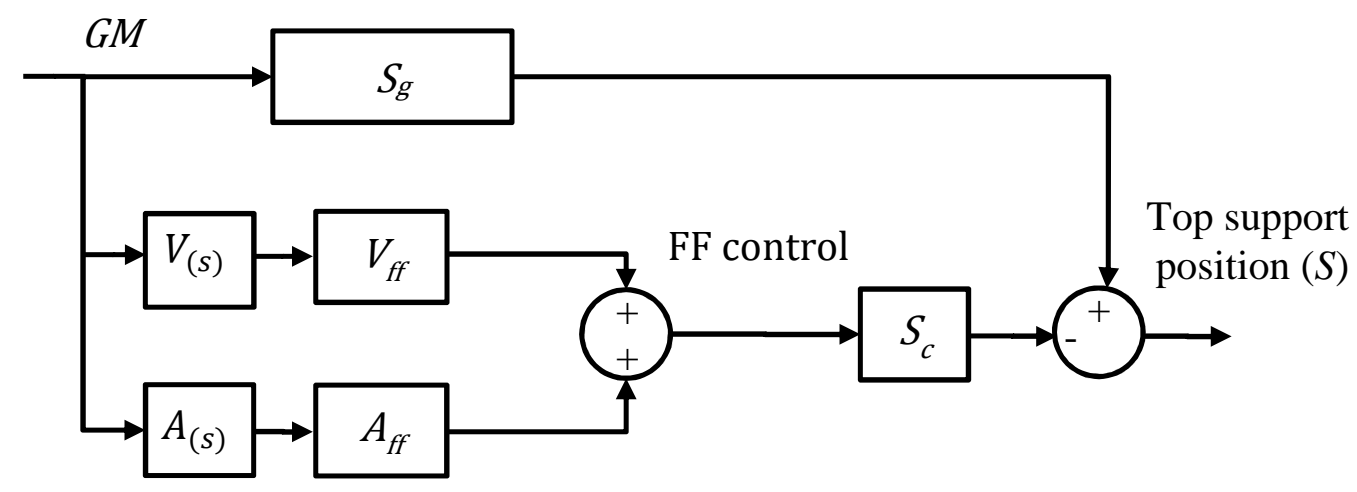

Figure 8. Block diagram of FF controls.

The velocity FF controller $V_{f f}$ is given by:

$$
V_{f f}=\hat{V}^{-1} \hat{S}_{c}^{-1} \hat{S}_{g} F_{v} .
$$

Where $F_{v}$ represents high and low pass filters as well as some extra poles to obtain proper transfer function and upper $\wedge$ denotes the identification of the corresponding transfer function. In the same way, the acceleration FF controller $A_{f f}$ is given by:

$$
A_{f f}=\hat{A}^{-1} \hat{S}_{c}^{-1} \hat{S}_{g} F_{a}
$$

Where $F_{a}$ plays the same role as $F_{v}$. The efficiency of FF controllers is nevertheless limited by imperfections and modeling errors. The control has therefore been extended with two feedback (FB) controllers that have been added to the control scheme, see Figure 9. 


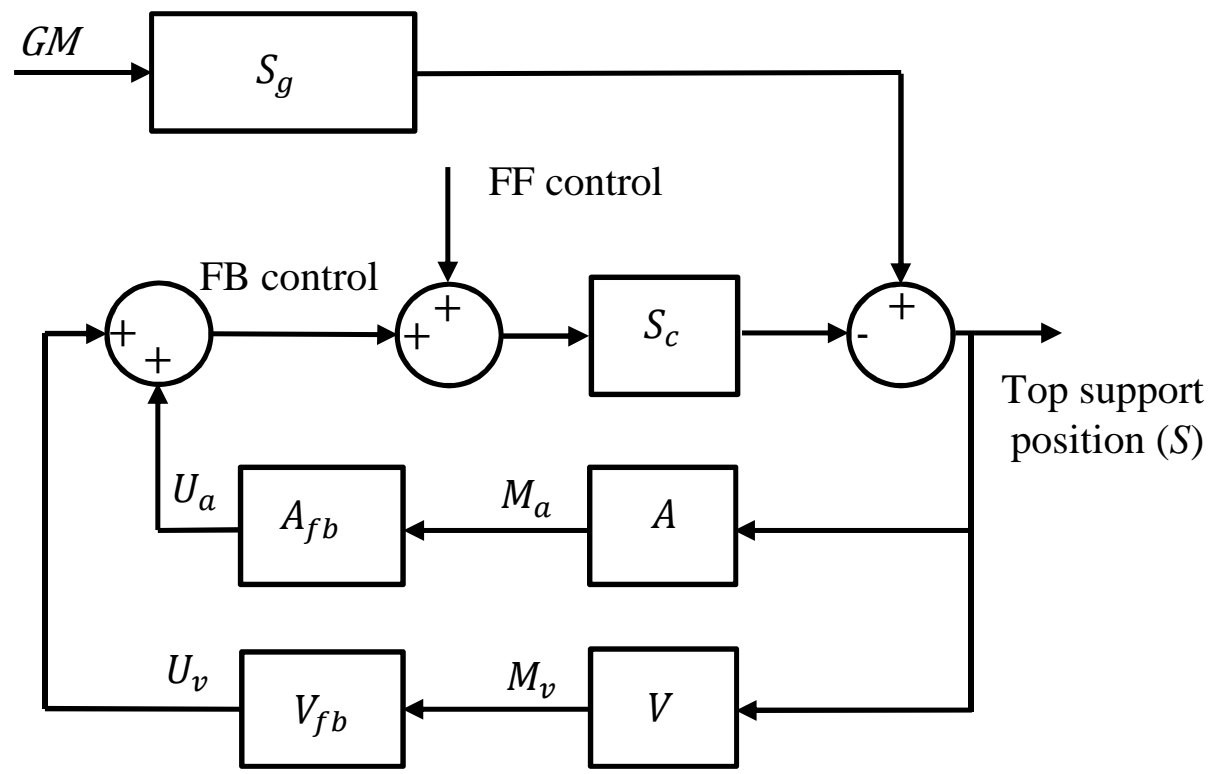

Figure 9. Block diagram of feedback controls.

The loop with the velocity sensor includes 3 derivatives and the loop with the accelerometer sensor includes 2 derivatives. The two controllers $A_{f b}$ and $V_{f b}$ are adjusted using loop shaping of the Nichols plot. Both controllers are computed with the following constraints on the open-loop:

- max gain close to $25 \mathrm{~dB}$,

- low-frequency $0 \mathrm{~dB}$ cross below $1 \mathrm{~Hz}$,

- high-frequency 0dB cross above $100 \mathrm{~Hz}$.

The FF and the FB controls are then added together, and lead to the theoretical attenuation given in Figure 10: 


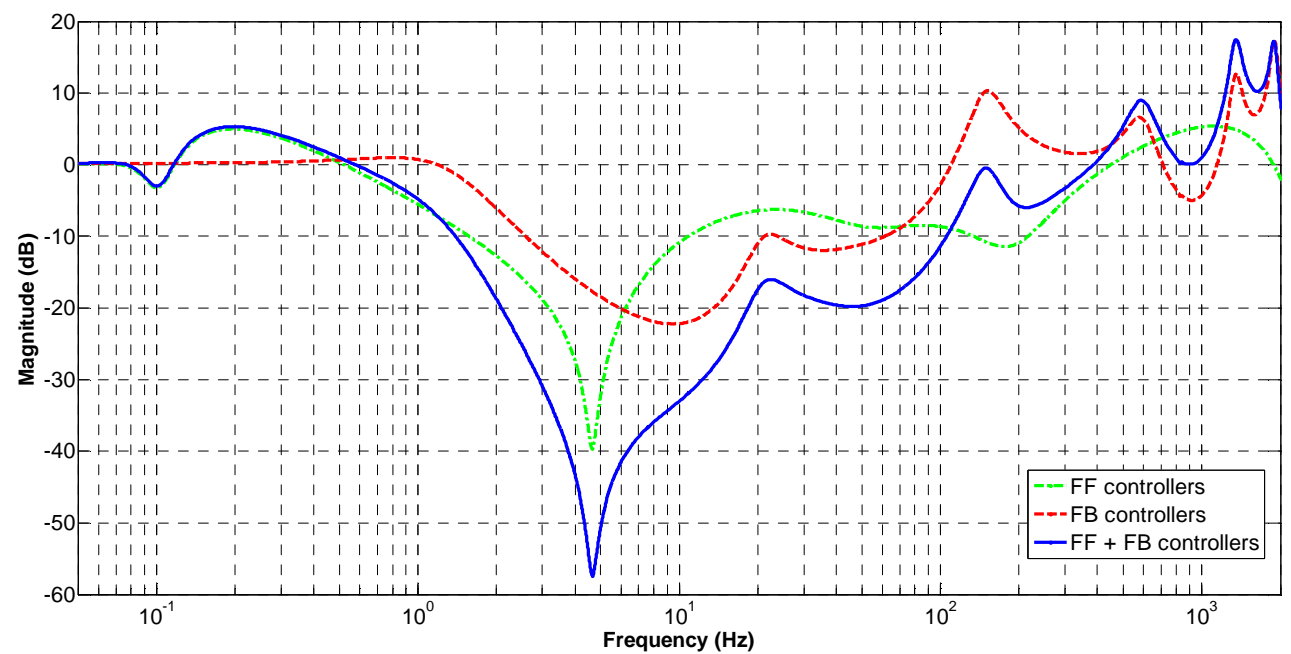

Figure 10. Theoretical attenuation of the whole control compared to FF and FB only.

\section{Noise considerations}

The obtained attenuation given in Figure 10 is ideal but the experimental one is limited by the presence of noise. Indeed, due to Bode's theorem, a high attenuation at low frequency increases the noise effect at high frequency. Hence, the controller settings must take into account all the noise sources discussed in section "Acquisition chain, sensors and analog converter noises". Figure 11 summarizes the noises that affect the output $S$.

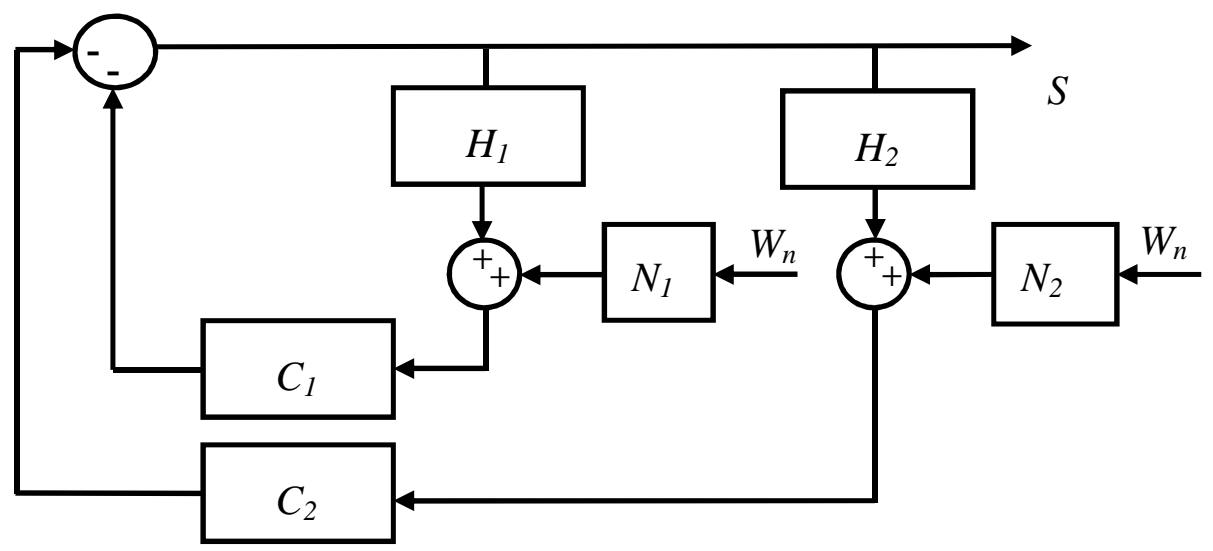

Figure 11. Noise sources.

$W_{n}$ through noise model $N_{1}$ or $N_{2}$ represents any of the noise sources (sensors, ADC and DAC) for the velocity and the acceleration loops, $C_{1}, C_{2}, H_{1}$ and $H_{2}$ are the transfer 
functions after and before the noise sources, $S$ is the signal affected by the noises.

For example, for the velocity sensor noise, we have:

$$
\begin{gathered}
C_{1}=S_{c} V_{f b}, \\
H_{1}=V, \\
N_{1}=N_{v} .
\end{gathered}
$$

Inside the bandwidth of the loops where $C_{1} H_{1} \gg 1$ and $C_{2} H_{2} \gg 1$ then:

$$
S=\frac{N_{1}}{H_{1}}+\frac{N_{2}}{H_{2}} \text {. }
$$

Outside the bandwidth of the loops where $C_{1} H_{1} \ll 1$ and $C_{2} H_{2} \ll 1$ then:

$$
S=C_{1} N_{1}+C_{2} N_{2} \text {. }
$$

Inside the bandwidth of the loops, high gains are needed for $H_{1}$ and $H_{2}$ and outside it is important that $C_{1}$ and $C_{2}$ have a low gain. This has been taken into account for the controller structure design and the needed performances.

Concerning the noises introduced by the FF controllers, they can easily be introduced using $N_{1}$ or $N_{2}$. The analytical effect of all noises (i.e. total equivalent output noise) is shown in Figure 12 and compared to a simulation plot of the top support motion obtained with the proposed control framework.

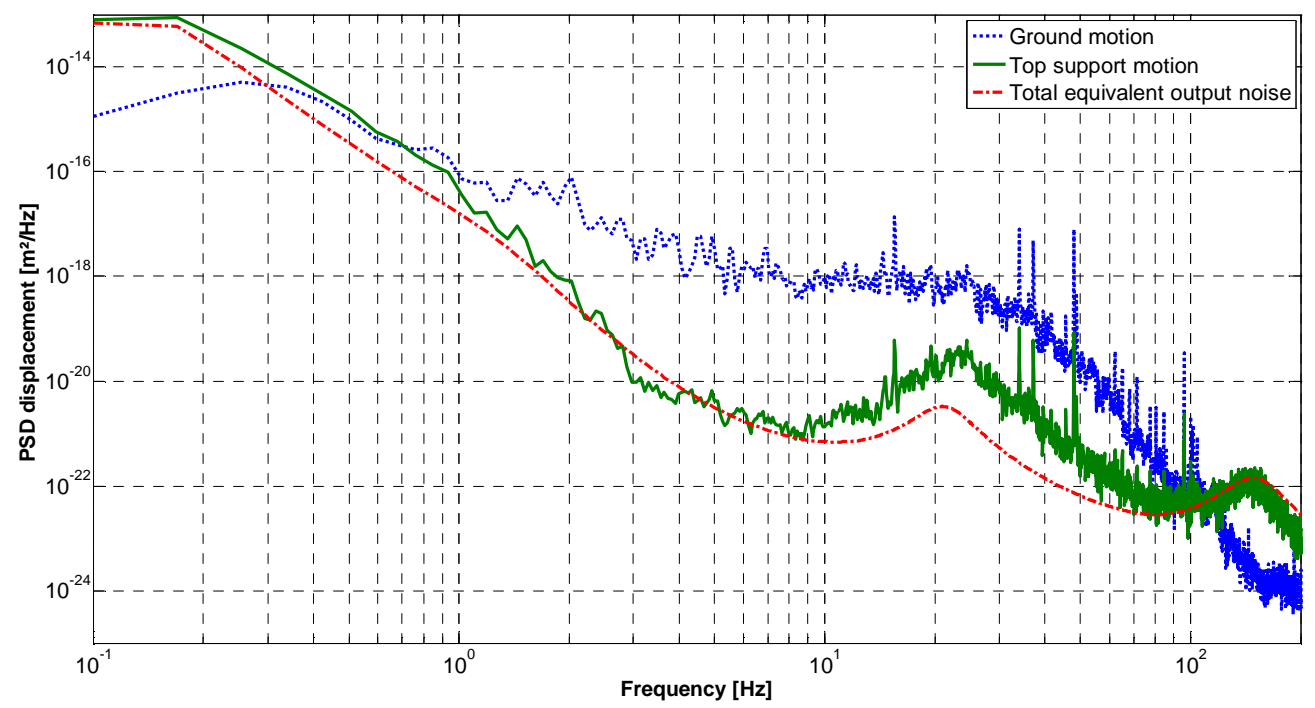

Figure 12. Effect of all noises on the output.

At low and high frequencies, Figure 12 shows that it is not possible to reduce further the support displacement due to the different noises in the control scheme where the top support motion is at the same level as the noises. An improvement can be expected around $20 \mathrm{~Hz}$. Figure 12 also shows that the main limitation of the attenuation 
is due to the different noise sources.

Based on this control scheme, a simulation program using MATLAB has been used in order to help define the two controllers:

- low and high pass filters for the FF controllers,

- loop-shaping of the FB controllers,

- best result for a given set of sensors

One of the main limitations being the sensor noise, this simulation program helps us define the sensor characteristics needed (noise characteristics and transfer function) for a given performance keeping in mind the need for reasonable costs. This is discussed in section "Results and improvements".

\section{TEST BENCH}

\section{Experimental setup}

The whole setup is shown in Figure 13. Sensor signals have been filtered using a real time 8th order Butterworth $20 \mathrm{kHz}$ low pass filter and amplified to fit optimally in the range $+/-5 \mathrm{~V}$ of the ADC channels.

All controllers are implemented using a digital scheme. For the controller discretization, the delta operator has been used (Goodwin et al., 1992) with a sampling period of $50 \mu \mathrm{s}$. The delta operator is useful in the presence of slow and fast dynamics and very small sampling period that could lead to bad numerical conditioning.

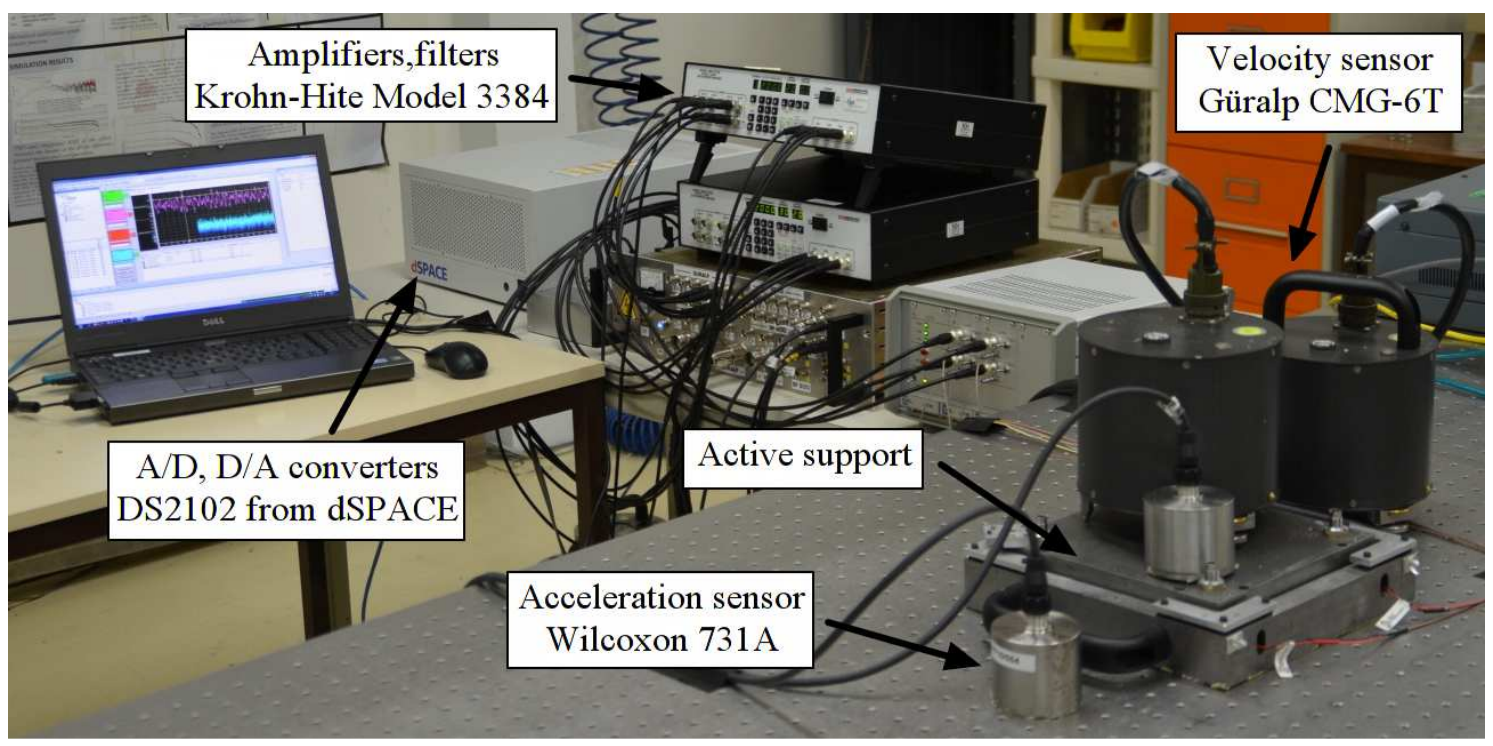

Figure 13. Experimental setup. 


\section{Results}

For a better understanding of the results, we will distinguish between the "real" support displacement as obtained through the control process, and the "observed" support displacement as measured by the sensors. The objective is to come as close as possible to the "real" displacement. The sensors being more or less accurate according to the frequency range and the sensor type, as shown in Figure 7, the "observed" PSD of the support motion has been reconstituted by taking the PSD at frequencies where the sensors are less noisy, that is (velocity sensors at low frequency and accelerometers at higher frequency):

$$
\widehat{P S D_{S}}=\left[P S D_{M_{v} \widehat{V}^{-1}}(0-16 \mathrm{~Hz}) ; P S D_{M_{a} \hat{A}^{-1}}(16 \mathrm{~Hz}-\infty)\right] .
$$

The experimental attenuation, compared to the simulation is given in Figure 14. The theoretical attenuation, corresponding to the "real" values that can be obtained by the system is also plotted on the figure.

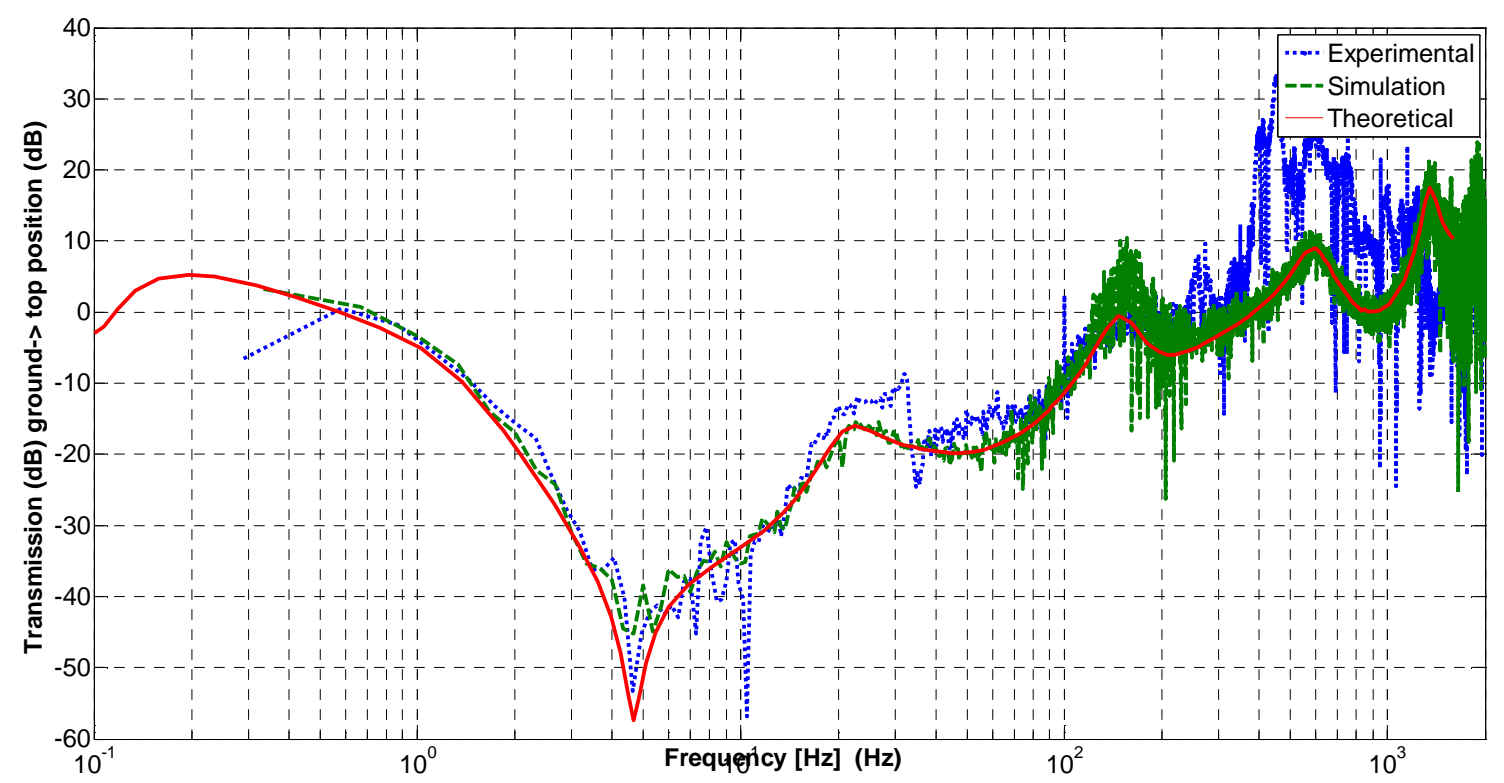

Figure 14. Theoretical attenuation and experimental attenuation compared to simulation.

The experimental results show a really good fit between theory and experiment. Albeit the obtained attenuation is an important criterion, the displacement RMS remains the reference. This leads to the experimental support motion RMS given in Figure 15. It is nevertheless not possible to reconstruct the GM outside the operating range of the sensors (i.e. $\mathrm{f} \in[1.5,200] \mathrm{Hz}$ ), where the real PSD should obviously be lower. 


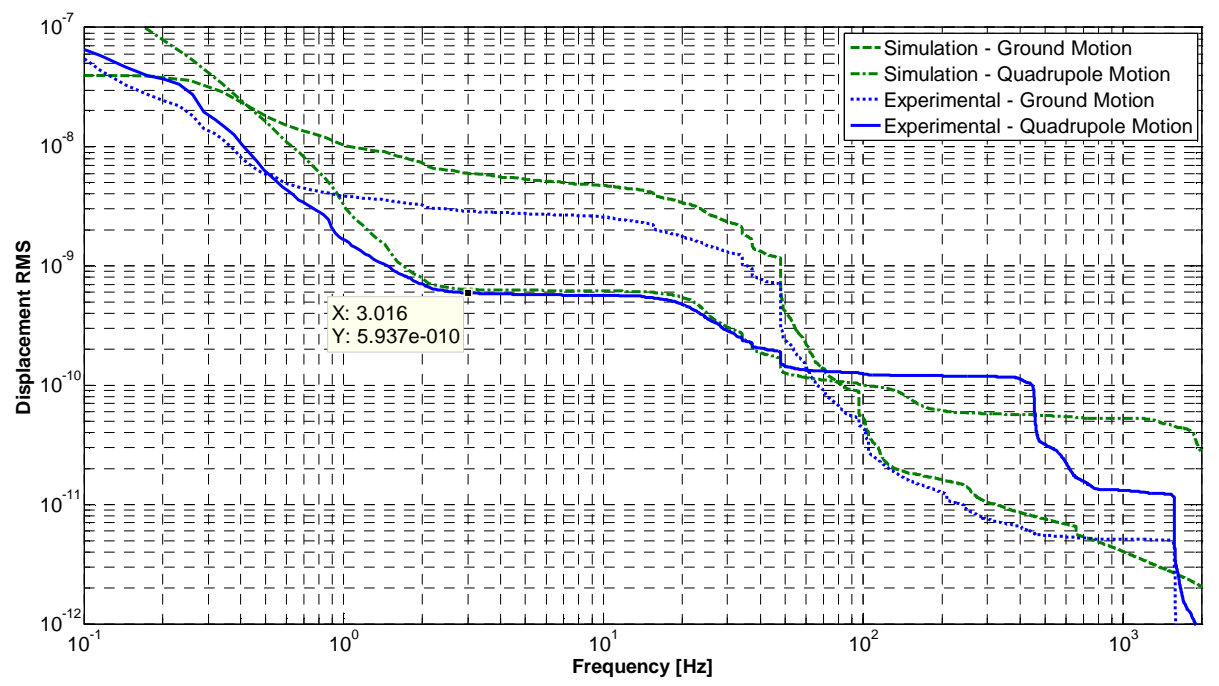

Figure 15. RMS comparison.

\section{RESULTS AND IMPROVEMENTS}

Concerning the ML, the needed performances of $1.5 \mathrm{~nm}$ at $1 \mathrm{~Hz}$ were reached with the proposed control strategy. Concerning the IP, the RMS is three times the needed performances. In order to obtain an RMS of $0.15 \mathrm{~nm}$ at $4 \mathrm{~Hz}$, the noises need to be further reduced. Figure 16(a) shows the sensors and hardware limitations;

(a)

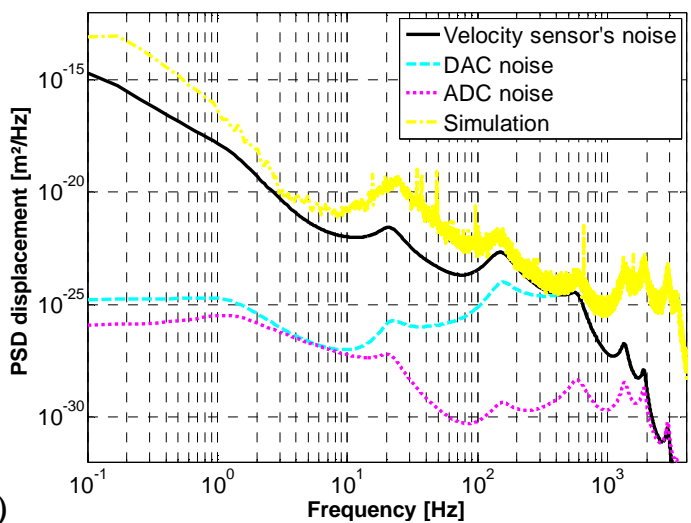

(b)

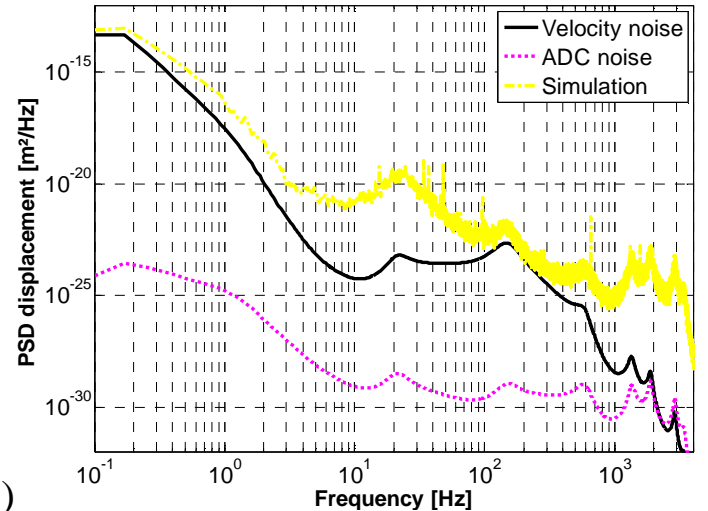

Figure 16. (a) Noises of FB velocity sensor, (b) Noises of FF velocity sensor.

The velocity sensors introduce too much noise at low and at high frequency. New commercial sensors have to be tested or a complete original design could be considered in order to increase the loop gain and then the system attenuation. The accelerometer has been used to reach higher frequencies, but its delay limits its usability in this range of frequencies, a new accelerometer has to be introduced in the control. Above $400 \mathrm{~Hz}$, 
the seismic attenuation is limited by the D/A converter noise. As the simulation and the experiment have similar behavior, it is possible to use the simulation results to find the new sensor and D/A characteristics needed to increase the system's performance further.

\section{CONCLUSION}

This study attempts to solve one of the most critical technical aspects of the future CLIC particle collider. In this prospect, a dedicated control strategy for ground motion mitigation is detailed. Based on a dedicated interpretation of classical loop shaping control design methods for controller tuning, the innovation consists in using sensors not intended for control at the sub-nanometer scale. Furthermore, the original control strategy consists in the cumulative action of acceleration and velocity feedforward control combined with feedback loops. In our approach, the Matlab/Simulink simulation results of the control are also compared with the real time experimental results. Theoretical results match the real time results with a small deviation, which is due to model imperfections and the limitation of the D/A converter resolution. The performance of the control, defined by the ratio RMS_GM/RMS_QM is about 5 at $4 \mathrm{~Hz}$ and 2.5 at $1 \mathrm{~Hz}$, comparable to the best stabilization strategy performances presented in Table 1. The results lead to a RMS displacement of the top support of about $1.5 \mathrm{~nm}$ at 1 $\mathrm{Hz}$ and $0.6 \mathrm{~nm}$ at $4 \mathrm{~Hz}$. Specifications are reached concerning the ML (RMS_QM (1) < $1.5 \mathrm{~nm}$ ) but not for the IP (RMS_QM $(4)<0.15 \mathrm{~nm}$ ). Possible ways for improving performance in GM mitigation are outlined. The main limitation concerns the sensors' noise, thus ongoing research efforts concentrate on sensors with better performances for this dedicated study with the help of the validated simulation program. Another avenue of improvement concerns the ADC resolution which induces limitations at high frequency. The next step will be to consider a representative heavy quadrupole on top of the support to address additional specifications.

\section{ACKNOWLEDGMENTS}

The authors wish to express their thanks to the European Commission for funding this research under the seventh framework program (project EuCARD, grant agreement no.227579).

\section{NOTE}

1. KEBE Scientific Instruments, Schwingungsmesser SMK-1 manual, Halstenbek 1994.

2. OYO Geospace Inc.

3. Stanford Linear Accelerator Center.

4. CEDRAT Group. 


\section{REFERENCES}

Artoos K, Collette C, Esposito M, et al. (2011) Modal analysis and measurement of water cooling induced vibrations on a CLIC main beam quadrupole prototype. Proceedings of International Particle Accelerator Conference (IPAC 2011). San Sebastian,

Balik G, Badel A, Bolzon B, et al. (2010) Stabilization study at the sub-nanometer level at the interaction point of the future Compact Linear Collider. Proceedings of Mechatronics (MECHATRONICS 2010). Yokohama, Japan.

Balik G, Brunetti L, Deleglise G, et al. (2011) Interaction point feedback design and integrated simulations to stabilize the CLIC final focus. Proceedings of International Particle Accelerator Conference (IPAC 2011).

Caron B, Balik G, Brunetti L and Jeremie A (2012) Vibration control of the beam of the future linear collider. Control Engineering Practice. Elsevier 20(3): 236-247. Available at:http://linkinghub.elsevier.com/retrieve/pii/S0967066111002334.

CERN collaboration (2012) A multi-TEV linear collider based on CLIC technology. .

Collette C, Janssens S, Artoos K, et al. (2011) Nano-motion control of heavy quadrupoles for future particle colliders: An experimental validation. Nuclear Instruments and Methods in Physics Research Section A: Accelerators, Spectrometers, Detectors and Associated Equipment. Elsevier 643(1): 95-101. Available at:http://linkinghub.elsevier.com/retrieve/pii/S0168900211007662.

Frisch J, Chang A, Decker V, et al. (2004) Vibration Stabilization of a Mechanical Model of a X-Band Linear Collider Final Focus Magnet. Proceedings of LINAC 2004. Lübeck, Germany, 684-686.

Frisch J, Decker V, Doyle E, et al. (2004) Development of a Non-Magnetic Inertial Sensor for Vibration Stabilization in a Linear Collider. Proceedings of LINAC 2004. Lübeck, 681-683.

Gaddi A, Gerwig H, Ramos F and Siegrist N (2012) Mechanical design of a pre-isolator for the CLIC final focusing magnets. .

Goodwin GC, Middleton RH and Poor H V (1992) High-speed digital signal processing and control. Proceedings of the IEEE. IEEE 80(2): 240-259. Available at:http://ieeexplore.ieee.org/iel1/5/3491/00123294.pdf?arnumber=123294.

Janssens S, Artoos K, Collette C, et al. (2011) Stabilization and positioning of CLIC quadrupole magnets with sub-nanometer resolution. Proceedings of ICALEPCS 2011. Grenoble, France, 74-77.

Montag C (1996) Active stabilization of mechanical quadrupole vibrations for linear colliders. Nuclear Instruments and Methods in Physics Research Section A: Accelerators, Spectrometers, Detectors and Associated Equipment. Elsevier 378(3): 369-375. Available at:http://www.sciencedirect.com/science/article/pii/0168900296004512.

Pfingstner J, Schulte D and Hofbaur M (2011) SVD-based filter design for the trajectory feedback of CLIC. IPAC 2011, the second International Particle Accelerator Conference. San Sebastián, Spain, 511-513. Available at:http://accelconf.web.cern.ch/AccelConf/IPAC2011/papers/mopo014.pdf.

Preumont A, Franc A and Bossens F (2002) Force feedback versus acceleration feedback in active vibration isolation. Journal of Sound and Vibration 257: 605-613.

Sarajlic E, Berenschot JW, Krijnen GJM and Elwenspoek MC (2003) Low volume, large force (>1mN) and nanometer resolution, electrostatic microactuator for low displacement applications. Nanotech 2003. Available at:http://doc.utwente.nl/46519/1/Sarajlic03low.pdf.

The CMS Collaboration (2008) The CMS experiment at the CERN LHC. Journal Of Instrumentation. IOP PUBLISHING LTD 3(08): S08004-S08004. Available at:http://stacks.iop.org/17480221/3/i=08/a=S08004?key=crossref.fc7f4422618a075830e017d1d930a35c.

The NLC Design Group (1996) Zeroth-order design report for the next linear collider. , 1005, Ground Motion : Theory and Measurement.

Tjepkema D, van Dijk J and Soemers HMJR (2012) Sensor fusion for active vibration isolation in precision equipment. Journal of Sound and Vibration. Elsevier 331(4): 735-749. Available at:http://linkinghub.elsevier.com/retrieve/pii/S0022460X11007656.

Virdee TS (2010) The LHC project: The accelerator and the experiments. Nuclear Instruments and Methods in Physics Research Section A. Elsevier 623(1): 1-10. Available at:http://linkinghub.elsevier.com/retrieve/pii/S0168900210004092. 